InIFolPharm

Fortgeschrittenes hepatozelluläres Karzinom

\title{
Effektive Second-Line-Therapie mit Met-Inhibition
}

\section{Der Met-Inhibitor Tivantinib verlängert die Zeit bis zur Tumorprogression. Vor allem Patienten mit hoher Expression des Met-Rezeptors im Tumor- gewebe profitieren von der Therapie.}

Ein überaktivierter Met-Signalweg beim hepatozellulären Karzinom (HCC) ist mit einer ungünstigen Prognose assoziiert.

In einer randomisierten Studie zeigte sich nun, dass Patienten mit hoher MetExpression des HCC von der zielgerichteten Therapie mit dem Met-Inhibitor Tivantinib profitieren können, erläuterte Bruno Daniele, Benevento, Italien. In der Studie wurden 107 bereits vortherapierte Patienten mit nicht resezierbarem HCC im Verhältnis 2:1 randomisiert mit Tivantinib oder Placebo behandelt [Rimassa L et al. J Clin Oncol. 2012;30(Suppl):4006].

Die mediane Zeit bis zur Progression war in der Intention-to-treat-Gruppe unter Tivantinib signifikant länger (6,9 vs. 6,0 Wochen unter Placebo; Hazard Ratio [HR] 0,64; $\mathrm{p}=0,04)$. Bei Patienten mit hoher Met-Expression im Tumorgewebe be- trug die mediane Zeit bis zur Progression sogar 11,7 Wochen unter Tivantinib (vs. 6,1 Wochen unter Placebo, HR 0,43; p = 0,03). Zudem ergab sich anders als in der Intention-to-treat-Gruppe auch beim medianen Gesamtüberleben ein Vorteil zugunsten von Tivantinib (7,2 vs. 3,8 Monate; HR 0,38; $p=0,01$ ).

Zudem erwies sich die Met-Expression im Tumorgewebe als prognostischer Faktor: Das Gesamtüberleben bei hoher MetExpression war bei den mit Placebo behandelten Patienten mit 3,8 Monaten deutlich schlechter als bei geringer MetExpression (9,0 Monate). Annemarie Musch

Pressekonferenz: „Tivantinib - A Promising Therapy in the Daiichi Sankyo Oncology Pipeline" im Rahmen des ESMO-Kongresses, Wien, 29.09.2012; Veranstalter: Daiichi Sankyo

\section{Hoffnungsvolle Ansätze für fortgeschrittene Erkrankungen}

\author{
Der Multi-Tyrosinkinase-Inhibitor \\ Lenvatinib zeigt in verschiedenen \\ Indikationen vielversprechende \\ Ergebnisse in Phase I und II.
}

Zielstrukturen des oralen TyrosinkinaseInhibitors Lenvatinib sind u.a. Rezeptoren unterschiedlicher Wachstumsfaktoren sowie die Rezeptoren RET und KIT. Ergebnisse verschiedener Phase-I- und -II-Studien mit Lenvatinib in unterschiedlichen Indikationen sind vielversprechend. So lag das vorläufige mediane Gesamtüberleben bei Patienten mit fortgeschrittenem hepatozellulären Karzinom bei 13,9 Monaten, die Zeit bis zur Progression betrug 7,5 Monate. 46 Patienten hatten Lenvatinib in einer Dosierung von $12 \mathrm{mg} / \mathrm{Tag}$ eingenommen [Ike- da K et al. Ann Oncol. 2012;23(Suppl 9):abstr. 737P]. An Nebenwirkungen waren bei Tyrosinkinase-Inhibitoren nicht ungewöhnliche Ereignisse wie Hypertension, palmare/plantare Erythrodysästhesie, Thrombopenie, Fatigue oder Proteinurie zu beobachten; neue Toxizitäten traten nicht auf.

Bei Patienten mit rezidivierendem Glioblastoma multiforme und Progression nach Gabe von Bevacizumab lag die 6-Monats-Gesamtüberlebensrate unter Therapie mit Lenvatinib bei $28 \%$; das mediane Gesamtüberleben lag bei 4,1 Monaten. Die progressionsfreie Überlebenszeit betrug 1,9 Monate [Reardon DA et al. Ann Oncol. 2012;23(Suppl 9):abstr. 417PD]. red

Nach Informationen von Eisai Neue Zulassung bei
Lymphom-Therapie

Die Europäische Kommission hat Ende Oktober die Zulassung für Brentuximab Vedotin (Adcetris ${ }^{\circledast}$ ) in zwei Indikationen erteilt. Das AntikörperWirkstoff-Konjugat kann bei erwachsenen Patienten mit rezidiviertem oder refraktärem CD-30-positiven Hodgkin-Lymphom eingesetzt werden, wenn eine autologe Stammzelltransplantation durchgeführt wurde oder mindestens zwei Vortherapien erfolgt sind und eine autologe Stammzelltransplantation bzw. eine Mehrfach-Chemotherapie nicht infrage kommen. Die zweite Indikation erstreckt sich auf Erwachsene mit rezidiviertem oder refraktärem systemischem anaplastischen großzelligen Lymphom (sALCL). Die Zulassung, so Andreas Engert, Köln, kennzeichnet einen wichtigen Fortschritt bei der Behandlung dieser seltenen Neoplasien, die rezidivieren oder refraktär sind und für die bisher nur begrenzte Therapieoptionen zur Verfügung standen. red

Nach Informationen von Takeda

\section{Aktuelle Richtlinien für GIST}

Die European Society for Medical Oncology (ESMO) aktualisierte im September ihre Leitlinien zur Diagnose, Therapie und Nachsorge von gastrointestinalen Stromatumoren (GIST). Bei Patienten mit primär operablen GIST und hohem Rezidivrisiko empfehlen die Experten als Standard nun eine dreijährige adjuvante Therapie mit Imatinib $\left(\right.$ Glivec $\left.^{\bullet}\right)$. Grundlage sind Ergebnisse der skandinavisch-deutschen Studie SSGXVIII/AIO [Joensuu $\mathrm{H}$ et al. JAMA 2012;307(12):1265-72]. Dabei konnten Daten von 397 Patienten nach kompletter Resektion eines c-KIT-positiven GIST mit hohem Rezidivrisiko ausgewertet werden. Fünf Jahre nach Therapiebeginn waren nach 36-monatiger Imatinib-Therapie $65,6 \%$ rezidivfrei, nach zwölfmonatiger Behandlung nur 47,9\%. Auch die Gesamtüberlebensrate war mit 92,0 vs. $81,7 \%$ unter der dreijährigen Behandlung deutlich günstiger. red

Nach Informationen von Novartis Pharma 